\title{
Ovarian tumors in pregnancy
}

Keywords: adenexal masses functional tumors, fetal maturity, asymptomatic masses

\section{Opinion}

Ovarian tumors are uncommon yet a serious complication of pregnancy. Their incidence has been quoted to be between $1 / 80$ to $1 / 640$ live births. They pose a challenge because the gravid uterus may prevent adequate palpation of the ovarian mass. Most ovarian enlargements during pregnancy are follicular or corpus luteal cyst which regresses as pregnancy advances. Functional cysts more than $6 \mathrm{cms}$ are rare.

Apart from increase in size, complications like torsion, hemorrhage, infection and rupture may occur in the tumor during pregnancy. The most serious but uncommon complication of ovarian tumors is malignancy. Because of the rarity of ovarian tumors complicating pregnancy, their characteristics, expectant management and operative interventions during pregnancy continue to be a dilemma. Surgical removal of ovarian tumor during pregnancy was first reported by Burd in 1846 .

They are rarely symptomatic during first trimester and are discovered on routine palpation or ultrasound examination. Some advocate removal of the tumor before the onset of symptoms and state that outcome of pregnancy is better if laparotomy is done electively. Though laparotomy is done on emergency basis in symptomatic complicated cases, management in case of symptomatic patients change with period of gestation.

Women with ovarian mass in first trimester should be observed till 16weeks of pregnancy. This allows resolution of functional cysts, prevent excision of corpus luteal cysts, avoid risks of spontaneous abortion and avoid possible adverse effects of anesthetic drugs on the fetus.

All significant adenexal masses that persist after 16 weeks should be electively removed to avoid delay in diagnosis of possible malignancy and to avoid risk of complications like rupture, torsion, hemorrhage, infection, obstruction of labour.
Volume 2 Issue 4 - 2017

\section{Nupur Hooja \\ Department of Obstetrics \& Gynecology, S.M.S. Medical College \& Hospital, India}

Correspondence: Nupur Hooja, Department of Obstetrics \& Gynecology, S.M.S. Medical College \& Hospital, Jaipur ( Rajasthan) 3020I5,A-29, Lal Bahadur Nagar, Girdhar Marg, Malviya Nagar, Jaipur-302017, Rajasthan, India, Tel 09828025302 , Email nupurhooja@gmail.com

Received: May 28, 2017 | Published: May 29, 2017

Asymptomatic masses first detected in third trimester should be observed till fetal maturity. This avoids possibility of preterm labour and technical difficulties of laparotomy. Once the fetal maturity is attained, elective caesarian section with removal masses is recommended.

\section{Conclusion}

Ovarian tumors in pregnancy emphasize the routine use of ultrasound in all obstetric patients which can detect adenaxal masses in relatively asymptomatic patients. The evaluation and management of ovarian tumors complicating pregnancy is different from such tumors in non-pregnant women. When suspicion of malignancy is high, operative interventions should be undertaken earlier in gestation.

\section{Acknowledgements}

None.

\section{Conflict of interest}

Author declares that there is no conflict of interest. 